\title{
A prospective study evaluating impact on renal function following percutaneous nephrolithotomy using Tc99m ethylenedicysteine renal scan: Does multiplicity of access tracts play a role?
}

\author{
Ram Yadav, Samarth Agarwal, Satyanarayan Sankhwar, Apul Goel, Manoj Kumar, Manmeet Singh, Ruchir Aeron, \\ Vikas Kumar \\ Department of Urology, King George's Medical University, Lucknow, Uttar Pradesh, India
}

Purpose: A prospective study evaluating impact of percutaneous nephrolithotomy (PCNL) on renal function following PCNL using ethylenedicysteine $(E C)$ renal scan. Does multiplicity of access tracts play a role?

Materials and Methods: A prospective observational study was done and patients undergoing PCNL for renal calculi at our urology department were selected. Renal Tc99m EC scan was done pre-surgery and at 3 months follow-up. An assessment was done on decline in glomerular filtration rate (GFR) in postoperative period based on number of access tracts required.

Results: A total of 110 patients were enrolled in the study. The total number of punctures was 170 with 141 being supra-costal puncture and 29 infra-costal. The total number of single punctures were 60 and classified as Group I whereas patients with double puncture and triple puncture (40 and 10 patients, respectively) were classified as Group II. Mean postoperative split renal function (in Tc99m EC scans) of patients of Group I (40.93 $\pm 19.62 \%)$ was found to be higher than that of Group II (32.82 $\pm 16.98 \%)$. Mean change (decline) in GFR for single, double and triple tracts were $2.68 \mathrm{~mL} / \mathrm{min}, 3.80 \mathrm{~mL} / \mathrm{min}$, and $4.2 \mathrm{~mL} / \mathrm{min}$, respectively.

Conclusions: PCNL used for stone removal can improve renal function by eradicating obstruction; however, this procedure may itself negatively impact the functions of the targeted kidney. Our study showed post PCNL decrease in GFR which worsens with an increasing number of accesses.

Keywords: Glomerular filtration rate; Kidney; Nephrolithiasis; Percutaneous nephrolithotomy; Radioisotope renography

This is an Open Access article distributed under the terms of the Creative Commons Attribution Non-Commercial License (http://creativecommons.org/licenses/by-nc/4.0) which permits unrestricted non-commercial use, distribution, and reproduction in any medium, provided the original work is properly cited.

\section{INTRODUCTION}

Renal calculi pose major health issues in our society with lifetime prevalence of kidney stone disease estimated at $1 \%$ to $15 \%$ [1]. The incidence of urinary tract stone disease is increasing. According to the National Health and Nutrition Examination Survey, as of 2012, 10.6\% of men and 7.1\% of women in the United States are affected by renal stone disease, compared to just $6.3 \%$ of men and $4.1 \%$ of women that were affected in 1994 [1].

Received: 13 August, 2018 - Accepted: 20 October, 2018

Corresponding Author: Samarth Agarwal

Department of Urology, King George's Medical University, Chowk, Lucknow 226003, Uttar Pradesh, India

TEL: +91-9702288820, E-mail: rebellite@gmail.com

ORCID: https://orcid.org/0000-0003-1390-6488 
Numerous surgical interventions are utilized to treat nephrolithiasis including shockwave lithotripsy, ureteroscopy, percutaneous nephrolithotomy (PCNL), laparoscopic, robotic assisted and open surgery. Based on the guidelines set forth by the American Urological Association (AUA) [2], PCNLs should be the first treatment utilized for patients with large/staghorn calculi. As per European Association of Urology (EAU) guidelines, shock wave lithotripsy (SWL) is the procedure of choice for most renal stones up to $15 \mathrm{~cm}$ except selected cases with unfavorable factors. PCNL is the choice for calculi larger than $1.5 \mathrm{~cm}$ without any absolute contraindication to the procedure [3]. The current indications for PCNL include large, hard infected stones, obstructionrelated stones, ESWL failures, and stones related with anatomical variations [4,5]

Though high success rate, PCNL is not without complications with a recent multi-centre study showing the overall complication rate of $20.5 \%$ [6]. In the setting of complex or multiple stones, more than one percutaneous access may be required for stone eradication, potentially increasing the risk of renal injury.

Renal functions are generally assessed with lab-based parameters such as estimated glomerular filtration rate (eGFR) or serum creatinine. Efficacy of these lab-based parameters can be greatly altered by patient factors including body mass index (BMI), renal functions at baseline, and race $[7,8]$.

Renal function after PCNL has also been assessed using nuclear medicine techniques. Various isotopes have been used for this function like Tc99m-mercaptoacetyltriglycine (MAG3), Tc99m-diethylene triamine pentacaetic acid (DTPA), Tc99m ethylenedicysteine (EC) and 131-Iodine labelled ortho Iodohippurate $(\mathrm{OIH})$ [9]. To determine the most relevant of the above, a study was done by Lima et al. [10], which concluded that Tc99m EC could substitute Tc99m-DTPA to evaluate patients with urinary tract dilation, with low cost and higher-resolution images.

A prospective study was done at our institution using Tc99m EC renography to better understand the effect of single versus multiple-access PCNL on individual renal function. We hypothesized that multi-tract PCNL should conceptually cause more renal injury than a single-tract procedure and therefore negatively affect split renal function.

\section{MATERIALS AND METHODS}

A prospective study was conducted at Department of Urology, King George's Medical University after approval by the Institutional Review Board and clearance by the Ethical Committee (approval number: 3689/ETHICS/RCELL18). After explaining the patients and their attendants about the study, a written informed consent was obtained by the patient or guardian (in case of minors) before enrollment into the study, regarding participation and follow-up visits.

A retrospective study was done from October 2015 to September 2017. Patients with renal calculi who opted for PCNL were included in the study. Patients found to have urinary tract or any other malignancy, pregnancy, known medical renal disease/compromised renal function (creatinine more than 2), anatomical/functional solitary kidney, bilateral renal calculi, patients with residual calculus more than 4 $\mathrm{mm}$ following PCNL and uncorrected bleeding disorders were excluded.

Radiological evaluation was done using ultrasonography kidney ureter bladder, intravenous pyelography (IVP) to evaluate anatomical and functional status of kidney as well as stone parameters. In case of raised serum creatinine noncontrast CT (NCCT) was done. Renal Tc99m EC scan was done pre-surgery and at 3 months post PCNL.

Preoperative urine culture was made sterile with appropriate antibiotics. Percutaneous nephrostomy tube was placed in patients with pyonephrosis and was operated after sterile urine culture. Prophylactic antibiotics were given in all patients. Intra operative data regarding stone access and stone retrieval were recorded in a detailed manner. The PCNL procedure was performed by single consultant in urology.

\section{Surgical techniques}

All patients received preop antibiotics. In lithotomy position, ureteric catheter $(4-\mathrm{Fr} / 5-\mathrm{Fr})$ was placed in the pelvicalyceal system and then patient was turned to prone position. The preferred puncture location was selected after studying the radiological imaging (IVP/NCCT/contrast enhanced computed tomography) in details. Percutaneous access was performed with the help of fluoroscopic guidance after obtaining air pyelogram. Tract was dilated using coaxial serial metal dilator over guide rod. In all patients the tracts were dilated to 28-Fr Amplatz sheath. Rigid nephroscope was used for all patients. Pneumatic lithotripsy (Swiss Lithoclast; Electro Medical Systems, Nyon, Switzerland) was used for stone fragmentation. Small fragments were removed using intra operative percutaneous caliceal irrigation. Extra puncture was performed if required.

Intra operatively, stone clearance was confirmed by nephroscope and fluoroscopy after the procedure. Double J stent was placed after complete removal of stone. Ureteric 
catheter was left in situ and fixed with per urethral catheter for those who need staged procedures. Nephrostomy tube (18-22-Fr) was placed after the procedures. Number of the nephrostomy tube was decided per operatively depending on the number of the access tracts and size of dilation needed. In multi tract cases, nephrostomy tubes were left in the tract if it was anticipated the tract would be used for a future procedure.

Stone free rate was defined as complete clearance or having insignificant residual $(<4 \mathrm{~mm})$ after the PCNL and evaluated by NCCT scan in the postoperative period. Perioperative complications like fever, septicaemia, hydro/ pneumothorax, severity of bleeding were evaluated for each patient and results tabulated. First follow-up at 2 weeks and thereafter follow-up was done at 3 months when EC renal scan was also performed.

\section{Statistical tools employed}

The statistical analysis was done using IBM SPSS Statistics ver. 21.0 software (IBM Co., Armonk, NY, USA). The values were represented in number (\%) and mean \pm standard deviation. Wilcoxon signed rank test was used to calculate change in serum creatinine, GFR and split function in the preoperative and postoperative period. Student t-test and chi-square tests were applied for calculations of mean changes between groups. A p-value less than 0.05 was considered significant.

\section{RESULTS}

\section{Study population}

A total of 132 patients were enrolled in this study. Out of 132 patients 8 patients excluded from study due to nonfunctioning of kidney in renal scan and 12 patients were lost to follow-up. In final analysis we included 110 patients. Out of 110 patients enrolled in the study, single puncture was required in 60 patients (54.5\%) which were classified as Group I while rest of the 50 patients (45.5\%) in whom 2 or more than 2 punctures were required were classified as Group II. Out of 110 patients right side calculi were in 61 patients (55.5\%) while rest 49 patients (44.5\%) were left sided.

\section{Demographic variables}

Overall mean age of study population was $34.65 \pm 13.23$ years. Among patients of Group I, 50.0\% were male and $50.0 \%$ were female while among patients of Group II, 74.0\% were male and only $26.0 \%$ were female.

\section{Radiological stone characteristics}

In Group I, most common site of stones was right side (68.3\%) followed by left side (31.7\%) while among patients of Group II, most common site was left side (60.0\%) followed by right side (40.0\%). Locations of stones in kidneys are also shown in Table 1.

Proportion of patients having single stone was statistically

Table 1. Demographic profile

\begin{tabular}{|c|c|c|c|}
\hline Variable & Group 1 (single puncture) & Group 2 (multiple puncture) & p-value \\
\hline Number of patients & 60 & 50 & - \\
\hline Age (y) & $34.65 \pm 13.23$ & $35.78 \pm 12.43$ & 0.828 \\
\hline Sex & & & 0.650 \\
\hline Male & $30(50.0)$ & $37(74.0)$ & \\
\hline Female & $30(50.0)$ & $13(26.0)$ & \\
\hline Body mass index $\left(\mathrm{kg} / \mathrm{m}^{2}\right)$ & $21.60 \pm 1.50$ & $21.62 \pm 1.51$ & 0.945 \\
\hline Stone surface area $\left(\mathrm{mm}^{2}\right)$ & $78.35 \pm 188.25$ & $643.00 \pm 296.77$ & 0.012 \\
\hline Side of stones & & & - \\
\hline Right & 41 & 20 & \\
\hline Left & 19 & 30 & \\
\hline Location of stones & & & - \\
\hline Pelvis & 52 & 48 & \\
\hline Upper calyx & 16 & 26 & \\
\hline Middle calyx & 3 & 18 & \\
\hline Inferior calyx & 12 & 27 & \\
\hline Hydronephrosis & & & 0.546 \\
\hline Mild & $25(41.7)$ & $16(32.0)$ & \\
\hline Moderate & $22(36.7)$ & $20(40.0)$ & \\
\hline Severe & $13(21.7)$ & $14(28.0)$ & \\
\hline
\end{tabular}

Values are presented as number only, mean \pm standard deviation, or number (\%). 
significantly higher in Group I as compared to Group II having single stone (65.0\% vs. $24.0 \%$ ), rest of the patients of both the groups had multiple stones. Difference in severity of hydronephrosis of patients of Group I and Group II was not found to be statistically significant $(\mathrm{p}=0.546)$.

Overall stone surface area was $498.65 \pm 276.22 \mathrm{~mm}^{2}$. Stone surface area was significantly high in Group II patients (643.00 \pm 296.77$)$ as compared to Group I (378.35 \pm 188.25$)$.

Urine culture of majority of patients $(n=96,87.3 \%)$ was sterile.

\section{Operative findings}

Out of 110 patients enrolled in the study, the total number of punctures was 170 . Out of the total no. Of punctures 141 were supra-costal puncture and 29 were infracostal puncture. The total number of single puncture was 60 , double puncture were 40 and triple puncture were in 10 patients. None of patient had 4 or more than 4 punctures.

Number of punctures in all the patients of Group I was one while in Group II ranged from 2-3 punctures. Proportion of patients with multiple stone was significantly higher in Group II (76.0\%) as compared to Group I (35.0\%).

Requirement of relook PCNL, requirement of blood transfusion and mean duration of removal of nephrostomy were all significantly higher in patients of Group II as compared to Group I (Table 2). According to modified Clavien Dindo Classification overall grade 2 complications was more common than grade 1 (30.0\% vs. $28.2 \%)$, but fever was most common (24.54\%) followed by blood transfusion
(21.8\%). None of the patients had grade 4/5 complications.

Mean postoperative serum creatinine of patients of Group II ( $1.24 \pm 0.44 \mathrm{mg} / \mathrm{dL})$ was found to be higher than that of Group I $(1.09 \pm 0.39 \mathrm{mg} / \mathrm{dL})$ but difference in postoperative mean serum creatinine levels of patients of Group I and Group II was not found to be statistically significant (Table 3).

Out of 110 patients enrolled in the study, mean postoperative split renal function (in Tc99m EC scans) of patients of Group I (40.93 $\pm 19.62 \%)$ was found to be higher than that of Group II (32.82 $\pm 16.98 \%)$ and this difference was found to be statistically significant.

Both mean preoperative and postoperative GFR of patients of Group I was found to be higher than that of Group II but difference in both was not statistically significant (Table 3).

On comparing changes in split renal functions among patients of Group I (1.25 $\pm 1.41 \%)$ and Group II (3.22 $\pm 1.15 \%)$, change was found to be significantly higher in Group II as compared to Group I. Overall change in split renal functions was $2.15 \pm 1.63$ units (5.6\%), which was also significant.

On univariate analysis, we did find a statistically significant difference in pre- and postoperative MAG3 renal function when stratification was performed for comorbidities such as diabetes mellitus, hypertension and other associated factors like guys stone score, degree of hydronephrosis and number of punctures which impacted renal function as shown in Table 4. A multivariable analysis using a logistic regression model was used to determine if

Table 2. Comparison of operative and postoperative findings between groups

\begin{tabular}{|c|c|c|c|c|c|}
\hline \multirow{2}{*}{ Variable } & \multirow{2}{*}{ Total $(n=110)$} & \multirow{2}{*}{ Group I $(n=60)$} & \multirow{2}{*}{ Group II $(n=50)$} & \multicolumn{2}{|c|}{ Statistical significance } \\
\hline & & & & $\chi^{2}$ & p-value \\
\hline Multiple number of stones & 59 & $21(35.0)$ & $38(76.0)$ & 18.436 & $<0.001$ \\
\hline Relook PCNL & 22 & $4(6.7)$ & $18(36.0)$ & 14.667 & $<0.001$ \\
\hline Blood transfusion & 20 & $4(6.7)$ & $16(32.0)$ & 11.766 & 0.001 \\
\hline Mean duration of removal of nephrostomy & $2.28 \pm 0.47$ & $2.05 \pm 0.29$ & $2.56 \pm 0.50$ & \multicolumn{2}{|c|}{$\begin{array}{l}\mathrm{t}^{\prime}=6.680 \\
\mathrm{p}<0.001\end{array}$} \\
\hline \multicolumn{6}{|l|}{ Grade 1 complications } \\
\hline Fever & 27 & $14(23.3)$ & $13(26.0)$ & 0.105 & 0.746 \\
\hline $\begin{array}{l}\text { Decrease urine output requiring diuresis } \\
\text { output required diuretics }\end{array}$ & 4 & $1(1.7)$ & $3(6.0)$ & 1.461 & 0.227 \\
\hline \multicolumn{6}{|l|}{ Grade 2 complications } \\
\hline Blood transfusions & 20 & $4(6.7)$ & $16(32.0)$ & 11.766 & 0.001 \\
\hline Febrile UTI & 13 & $8(13.3)$ & $5(10.0)$ & 0.291 & 0.590 \\
\hline \multicolumn{6}{|l|}{ Grade 3 complications } \\
\hline Urine leak $>24$ hours & 4 & $2(3.3)$ & $2(4.0)$ & 0.035 & 0.852 \\
\hline Pneumothorax & 1 & 0 & $1(2.0)$ & 1.211 & 0.271 \\
\hline
\end{tabular}

Values are presented as number only, number (\%), or mean \pm standard deviation. PCNL, percutaneous nephrolithotomy; UTI, urinary tract infection. 
Table 3. Comparison of pre- and postoperative serum creatinine, GFR and split renal function

\begin{tabular}{cccc}
\hline Variable & Group 1 (single puncture) & Group 2 (multiple puncture) & p-value \\
\hline Serum creatinine & & & 0.232 \\
Preoperative & $1.04 \pm 0.36$ & $1.12 \pm 0.42$ & 0.063 \\
Postoperative & $1.09 \pm 0.39$ & $1.24 \pm 0.44$ & 0.708 \\
GFR & & $33.58 \pm 17.74$ & 0.473 \\
Preoperative & $34.83 \pm 17.21$ & $29.70 \pm 18.26$ & 0.080 \\
Postoperative & $32.15 \pm 17.35$ & & 0.024 \\
Split renal function & & $36.04 \pm 16.94$ & $32.82 \pm 16.98$ \\
Preoperative & $42.18 \pm 19.08$ & & \\
Postoperative & $40.93 \pm 19.62$ & & \\
\hline
\end{tabular}

Values are presented as mean \pm standard deviation.

GFR, glomerular filtration rate.

Table 4. Univariate analysis of change in GFR (pre- and postoperative) with other variables

\begin{tabular}{|c|c|c|c|c|c|c|c|}
\hline \multirow{2}{*}{ Variable } & \multirow{2}{*}{ No. of patients } & \multirow{2}{*}{ Preoperative GFR } & \multirow{2}{*}{ Postoperative GFR } & \multirow{2}{*}{ Change } & \multirow{2}{*}{$\%$ change } & \multicolumn{2}{|c|}{ Paired t-test } \\
\hline & & & & & & $\mathrm{t}$ & p-value \\
\hline \multicolumn{8}{|l|}{ Comorbidities } \\
\hline Diabetes & 9 & $27.00 \pm 14.76$ & $22.11 \pm 14.86$ & $-4.89 \pm 1.45$ & -18.1 & -10.094 & $<0.001$ \\
\hline HTN & 7 & $34.43 \pm 16.76$ & $30.00 \pm 17.32$ & $-4.43 \pm 1.90$ & -12.9 & -6.519 & 0.001 \\
\hline $\mathrm{DM}+\mathrm{HTN}$ & 2 & $15.50 \pm 2.12$ & $11.50 \pm 3.54$ & $-4.00 \pm 1.41$ & -25.8 & -4.000 & 0.156 \\
\hline No comor & 92 & $35.37 \pm 17.61$ & $32.41 \pm 17.86$ & $-2.96 \pm 1.97$ & -8.4 & -14.422 & $<0.001$ \\
\hline \multicolumn{8}{|c|}{ Guy's stone score } \\
\hline 1 & 36 & $32.03 \pm 15.28$ & $29.22 \pm 15.85$ & $-2.81 \pm 1.55$ & -8.76 & -10.889 & $<0.001$ \\
\hline 2 & 42 & $39.31 \pm 17.70$ & $35.74 \pm 17.95$ & $-3.57 \pm 1.89$ & -9.09 & -12.253 & $<0.001$ \\
\hline 3 & 15 & $26.47 \pm 16.94$ & $23.13 \pm 16.75$ & $-3.33 \pm 2.58$ & -12.6 & -5.000 & $<0.001$ \\
\hline 4 & 17 & $33.41 \pm 18.92$ & $30.24 \pm 19.91$ & $-3.18 \pm 2.51$ & -9.51 & -5.226 & $<0.001$ \\
\hline \multicolumn{8}{|l|}{ Hydronephrosis } \\
\hline Mild & 41 & $38.85 \pm 14.22$ & $35.88 \pm 14.74$ & $-2.98 \pm 2.09$ & -7.66 & -9.110 & $<0.001$ \\
\hline Moderate & 42 & $33.71 \pm 17.43$ & $30.10 \pm 17.71$ & $-3.62 \pm 1.62$ & -10.7 & -14.457 & $<0.001$ \\
\hline Severe & 27 & $28.15 \pm 20.10$ & $25.15 \pm 20.36$ & $-3.00 \pm 2.34$ & -10.7 & -6.670 & $<0.001$ \\
\hline \multicolumn{8}{|c|}{ Number of puncture } \\
\hline 1 & 60 & $34.83 \pm 17.21$ & $32.15 \pm 17.35$ & $-2.68 \pm 1.84$ & -7.7 & -11.318 & $<0.001$ \\
\hline 2 & 40 & $35.20 \pm 17.56$ & $31.40 \pm 18.17$ & $-3.80 \pm 1.96$ & -10.8 & -12.238 & $<0.001$ \\
\hline 3 & 10 & $27.10 \pm 17.84$ & $22.90 \pm 17.93$ & $-4.20 \pm 2.25$ & -15.5 & -5.900 & $<0.001$ \\
\hline
\end{tabular}

Values are presented as number only or mean \pm standard deviation.

GFR, glomerular filtration rate; HTN, hypertension; DM, diabetes mellitus.

any of the following potential risk factors was associated with significant decline in GFR (>15\%): age, sex, diabetes, hypertension, and number of access tracts (one vs two/three). The independent risk factors identified as predictors of a deterioration in GFR of $>15 \%$ from baseline were elevated diabetes (coefficient, 3.85; $\mathrm{p}=0.025$ ), and number of access tracts (coefficient, 2.85; $\mathrm{p}=0.050$ )

\section{DISCUSSION}

PCNL is an established technique for treatment of renal calculi. The 2005 AUA and 2008 EAU guidelines on the management of staghorn calculi recommended PCNL as the first-line treatment for staghorn calculi, any renal stone that exceeds $20 \mathrm{~mm}$ in diameter, and lower calyceal stones [2,11].

Multiple or staghorn or complex stones may require more than one renal puncture during PCNL which may cause more renal parenchymal injury compare to single renal puncture. There are various studies done in past on this effect and showed divergent results. However most of these studies used lab-based parameters including the Modification of Diet in Renal Diseases (weight as variable) and Cockcroft-Gault equations (race as variable) formula, which are commonly used estimates of renal function [12]. 
These lab-based parameters are affected by patient's age sex, race, BMI, and base line renal parameters and alter the calculation of eGFR [12].

When patient is planned for PCNL on one side, the global creatinine based assessment is confounded by opposite normal kidney function. Nuclear renal scan provides a much more accurate assessment of individual renal functions. Previous studies used DMSA, DTPA, and MAG3 Tc99m renal scans to know the impact of PCNL on renal functions. In our study we used Tc99m EC (L, L-ethylenedicysteine) scan to know impact on renal functions.

Our study demonstrated that multi access PCNL had a trend towards decreasing GFR as the number of puncture increases although there was no association between two cohorts and serum creatinine levels.

In our study, mean age of patients was $34.65 \pm 13.23$ years which was slight lower compared to studies done by Fayad et al. [13], Gorbachinsky et al. [14], Moskovitz et al. [15], where reported mean age distribution was 39-53 years. This could be due to two third patients in our study were younger age group between 16-45 years and 7 patients were less than 15 years. Age groups in both the groups single versus multiple accesses were comparable in our study.

In this study, 67 patients $(60.9 \%)$ were male while 43 patients (39.1\%) were female. This high incidence of renal stones in males (15:1) is similar to that reported by Fayad et al. [13], and is in accordance to the reported high incidence of renal calculi in males [14]. BMI in our study population was $21.61 \pm 1.50$, which was comparable in both the groups.

The overall stone surface area was $498.65 \pm 276.22 \mathrm{~mm}^{2}$ and it was slightly higher than study done by Pérez-Fentes et al. [16] in which stone surface area was $356 \mathrm{~mm}^{2}$. This difference may be due to more no of patients with multiple renal calculi in our study compare to their study (51 vs. 17). The stone surface area was significantly high in Group II (multiple accesses) patients compared to Group I (643.00 \pm 296.77 vs. $378.35 \pm 188.25)$. Patients with multiple number of stones were also significantly high in multiple accesses group. PCNL tract dilatation in both the groups was 28-Fr.

In our study relook PCNL was required for complete clearance in 22 of patients (20\%) which is comparable to study done by Moskovitz et al. [15], they showed $90 \%$ stone free rate. Study done by Thomas et al. [17] showed stone clearance $62 \%$ overall and $30 \%-80 \%$ according to Guy's stone score. This is because of more no of patients with staghorn calculi in their study compare to our study ( $29 \%$ vs. $15 \%$ ).

\section{Comparison of pre- and postoperative renal functions: Tc99m EC scan}

The majority of patients showed decrease in split renal functions and decline in GFR after intervention in same renal unit. In both the groups significant decline in renal functions after intervention was observed. In Group I and Group II changes in pre and postoperative split renal functions were $1.25 \%$ and $3.22 \%$. Furthermore, there was a trend toward worsening renal function with an increasing number of accesses.

Similar results were noted by Gorbachinsky et al. [14], they shown significant $2.28 \%$ decrease in renal function based on mercaptoacetyltriglycine nuclear renogram results after PCNL of the affected kidney in patients with multiple accesses. Similar results also noted by Fayad et al. [13], that PCNL with multiple tracts carries a risk for adversely affecting renal function than single tracts. Chatham et al. [18] studied 19 patients with MAG3 renography after PCNL. At a median of 22 days after surgery they found $16 \%$ had a decrease in renal function, $37 \%$ had improvement and the remainder had no significant alteration. Compare to our study this study had only 19 patients and they did post PCNL renal scan which is earlier than our study (3 weeks vs. 3 months).

Because diabetes is more common in patients with kidney stones who develop CKD, it is likely that the coexistence of both situations would explain the increased risk of CKD in this kidney stone cohort. Ozden et al. [19] have found by multivariate analysis that DM was a significant marker for subsequent decline in GFR in patients undergoing PCNL. This association was also seen in our study.

As the number of punctures increases, there is increase in change (decline) in GFR after intervention. Mean change (decline) in GFR for single, double and triple were $2.68 \mathrm{~mL} /$ $\mathrm{min}, 3.80 \mathrm{~mL} / \mathrm{min}$, and $4.2 \mathrm{~mL} / \mathrm{min}$, respectively. In our study, renal function declined after surgery even in those with severe hydronephrosis. The drop was less than mild, but still significant. This is worth highlighting as urologists often debate the merits of PCNL in terms of improving renal function (i.e., someone presents with a large stone and split renal function of 15\%-is PCNL or nephrectomy the better option?). Based on our data it would suggest that it would be unlikely to see an improvement in function and perhaps a nephrectomy is the simpler option with a lower overall complication rate.

\section{Limitation of study}

Our study comprised of only 110 patients, with limited follow-up period of 3 months at a single center. The other 
limitation is the lack of metabolic evaluation and stone analysis in our study.

\section{CONCLUSIONS}

PCNL used for stone removal can improve renal function by eradicating obstruction; however, this procedure may itself negatively impact the functions of the targeted kidney. Our study showed post PCNL decrease in GFR which worsens with an increasing number of accesses. This may have significant impact in those with altered renal function or those who may require repetitive multi stage procedures. As such, attaining additional access during PCNL, especially in patients with concomitant renal insufficiency, should be considered carefully due to the profound impact on renal function and consequent impaired renal function recovery in this patient subset. Accurate assessment of this relationship may help urologists to guide surgical decisions, especially in patients with comorbidities associated with poor renal recovery following PCNL.

\section{CONFLICTS OF INTEREST}

The authors have nothing to disclose.

\section{ACKNOWLEDGMENTS}

I acknowledge the cooperation of residents of Urology Department of King George's Medical University and the faculty and residents of radiotherapy department who participated in data collection and evaluation of the patient. We also appreciate the commitment and compliance of the patient who reported the required data.

\section{REFERENCES}

1. Scales CD Jr, Smith AC, Hanley JM, Saigal CS; Urologic Diseases in America Project. Prevalence of kidney stones in the United States. Eur Urol 2012;62:160-5.

2. Preminger GM, Assimos DG, Lingeman JE, Nakada SY, Pearle MS, Wolf JS Jr; AUA Nephrolithiasis Guideline Panel. Chapter 1: AUA guideline on management of staghorn calculi: diagnosis and treatment recommendations. J Urol 2005;173:19912000.

3. Türk C, Petř́k A, Sarica K, Seitz C, Skolarikos A, Straub M, et al. EAU guidelines on diagnosis and conservative management of urolithiasis. Eur Urol 2016;69:468-74.

4. Skolarikos A, Alivizatos G, de la Rosette JJ. Percutaneous nephrolithotomy and its legacy. Eur Urol 2005;47:22-8.
5. Skolarikos A, de la Rosette J. Prevention and treatment of complications following percutaneous nephrolithotomy. Curr Opin Urol 2008;18:229-34.

6. Cho HJ, Lee JY, Kim SW, Hwang TK, Hong SH. Percutaneous nephrolithotomy for complex renal calculi: is multi-tract approach ok? Can J Urol 2012;19:6360-5.

7. Mahajan S, Mukhiya GK, Singh R, Tiwari SC, Kalra V, Bhowmik DM, et al. Assessing glomerular filtration rate in healthy Indian adults: a comparison of various prediction equations. J Nephrol 2005;18:257-61.

8. Poggio ED, Wang X, Greene T, Van Lente F, Hall PM. Performance of the modification of diet in renal disease and Cockcroft-Gault equations in the estimation of GFR in health and in chronic kidney disease. J Am Soc Nephrol 2005;16:459-66.

9. Taylor AT. Radionuclides in nephrourology, part 1: radiopharmaceuticals, quality control, and quantitative indices. J Nucl Med 2014;55:608-15.

10. Lima MC, de Lima ML, Pepe CF, Etchebehere EC, Santos AO, Amorim BJ, et al. Technetium-99m-L,L-ethylenedicysteine is more effective than technetium-99m diethylenetriamine penta-acetic acid for excluding obstruction in patients with pyelocalicinal dilation. Urology 2010;76:283-8.

11. Tiselius HG, Ackermann D, Alken P, Buck C, Conort P, Gallucci M; Working Party on Lithiasis, European Association of Urology. Guidelines on urolithiasis. Eur Urol 2001;40:362-71.

12. Levey AS, Inker LA, Coresh J. GFR estimation: from physiology to public health. Am J Kidney Dis 2014;63:820-34.

13. Fayad AS, Elsheikh MG, Mosharafa A, El-Sergany R, AbdelRassoul MA, Elshenofy A, et al. Effect of multiple access tracts during percutaneous nephrolithotomy on renal function: evaluation of risk factors for renal function deterioration. J Endourol 2014;28:775-9.

14. Gorbachinsky I, Wood K, Colaco M, Hemal S, Mettu J, Mirzazadeh $\mathrm{M}$, et al. Evaluation of renal function after percutaneous nephrolithotomy-does the number of percutaneous access tracts matter? J Urol 2016;196:131-6.

15. Moskovitz B, Halachmi S, Sopov V, Burbara J, Horev N, Groshar D, et al. Effect of percutaneous nephrolithotripsy on renal function: assessment with quantitative SPECT of (99m)TcDMSA renal scintigraphy. J Endourol 2006;20:102-6.

16. Pérez-Fentes D, Cortés J, Gude F, García C, Ruibal A, Aguiar P. Does percutaneous nephrolithotomy and its outcomes have an impact on renal function? Quantitative analysis using SPECTCT DMSA. Urolithiasis 2014;42:461-7.

17. Thomas K, Smith NC, Hegarty N, Glass JM. The Guy's stone score--grading the complexity of percutaneous nephrolithotomy procedures. Urology 2011;78:277-81.

18. Chatham JR, Dykes TE, Kennon WG, Schwartz BF. Effect of percutaneous nephrolithotomy on differential renal function 


\section{Yadav et al}

as measured by mercaptoacetyl triglycine nuclear renography. Urology 2002;59:522-5; discussion 525-6.

19. Ozden E, Mercimek MN, Bostanci Y, Yakupoglu YK, Sirtbas
A, Sarikaya S. Long-term outcomes of percutaneous nephrolithotomy in patients with chronic kidney disease: a singlecenter experience. Urology 2012;79:990-4. 